\title{
Preparation of 4-aryl- $\beta$-carboline-3-carboxamides by Suzuki-Miyaura cross-coupling applied to a 4-iodo precursor obtained by ortho- directed lithiation
}

\author{
Lisbet Xuarez-Marill and Robert H. Dodd* \\ Institut de Chimie des Substances Naturelles, UPR 2301, Centre National de la Recherche \\ Scientifique, Avenue de la Terrasse, 91198 Gif-sur-Yvette, France \\ E-mail: robert.dodd@icsn.cnrs-gif.fr
}

Dedicated to Professor Guy Quéguiner on the occasion of his 70th birthday

\begin{abstract}
The 4-iodo- $\beta$-carboline-3-carboxamide derivative $\mathbf{2}$ was obtained by ortho- directed metalation of the phenylcarboxamide precursor 5 using methyllithium and iodine followed by in situ $\mathrm{N}$ methylation with methyl iodide. Compound $\mathbf{2}$ reacted with a variety of aryl- and heteroarylboronic acids under classical Suzuki-Miyaura palladium-catalyzed coupling conditions to give the corresponding 4-aryl (7a-d, 7f) and 4-heteroaryl (7g, 7h) $\beta$-carboline-3carboxamides. While this reaction failed in the case of 3,4,5-trimethoxyphenylboronic acid, conditions were found $\left[\mathrm{Pd}(\mathrm{OAc})_{2}\right.$, dppf, $\mathrm{CsF}$ in dioxane-ethanol] which allowed formation of the coupled product $7 \mathbf{e}$ in high yield. The $N, N$-dimethylsulfamoyl protecting group was easily removed using a trifluoroacetic acid-trifluoromethanesulfonic acid mixture. Reductive amination of the 4-(2-formylphenyl)- $\beta$-carboline derivative $7 \mathbf{f}$ with benzylamine followed by sodium hydride- promoted cyclization provided the novel benzazepino- $\beta$-carboline derivative 9 .
\end{abstract}

Keywords: 4-Aryl- $\beta$-carboline, ortho- directed metalation, Suzuki coupling, benzazepino- $\beta$ carboline, palladium- catalyzed coupling

\section{Introduction}

$\beta$-Carbolines represent an important heterocyclic system, being found both as constituents of many natural products and in a number of pharmacologically active compounds. ${ }^{1,2}$ In particular, $\beta$-carboline-3-carboxylates and -carboxamides have been shown to possess high affinities for the benzodiazepine (e.g., Valium ${ }^{\circledR}$ ) binding site of the $\gamma$-aminobutyric acid (GABA) receptor of the central nervous system. ${ }^{3}$ Such compounds thus have potential as anti-epileptic, anxiolytic or mnemotonic drugs. However, while many $\beta$-carboline analogues have been synthesized which 
demonstrate potentially clinically useful profiles, none has found its way into use as a drug for humans. Thus, there is a need to find $\beta$-carboline-3-carboxylic acid derivatives which, while maintaining pharmacological activity, are more selective, more bio-available and/or less toxic.

The C-4 position of $\beta$-carboline-3-carboxylates is well known to accept a wide range of substituents allowing pharmacomodulation with little or no loss, and sometimes even remarkable increase, in receptor affinity. ${ }^{4,5}$ In this connection, we have previously described the selective introduction of halogen atoms (Br, I) at C-4 of phenyl $\beta$-carboline-3-carboxamide (1 and $\mathbf{2}$, Scheme 1) ${ }^{6-8}$ via ortho- directed lithiation/electrophilic substitution. ${ }^{9}$ Metalation of the C-4 position was successfully accomplished only when methyllithium was used as the lithiating agent. Furthermore, among the many possible $N$-9 $\beta$-carboline protecting groups, only the $N, N$ dimethylsulfamoyl group was found to be sufficiently stable under the lithiating reaction conditions. Both C-4 halogenated derivatives $\mathbf{1}$ and $\mathbf{2}$ were subsequently used to introduce other substituents using palladium-catalyzed coupling reactions. Thus, the 4-bromo-3-carboxamide 1 was converted ${ }^{7,8}$ into 4 -amino derivatives (3) using Buchwald conditions. ${ }^{10}$ However, the application of Stille- or Heck- type coupling reactions to the 4-iodo analogue of $\mathbf{1}$ required prior transformation of the secondary to a tertiary carboxamide in order to avoid complexation and deactivation of the palladium catalyst. ${ }^{8}$ Thus, these palladium-catalyzed reactions were successful starting from the 4-iodo-3- $N$-methyl- $N$-phenylcarboxamido- $\beta$-carboline $\mathbf{2}$, allowing introduction of unsaturated $\mathrm{C}-\mathrm{C}$ bonded substituents at C-4 (4). In an effort to provide further structural diversity, we now describe the preparation of 4-aryl- $\beta$-carboline-3-carboxamides by way of Suzuki-Miyaura cross-coupling reactions and modified versions thereof.
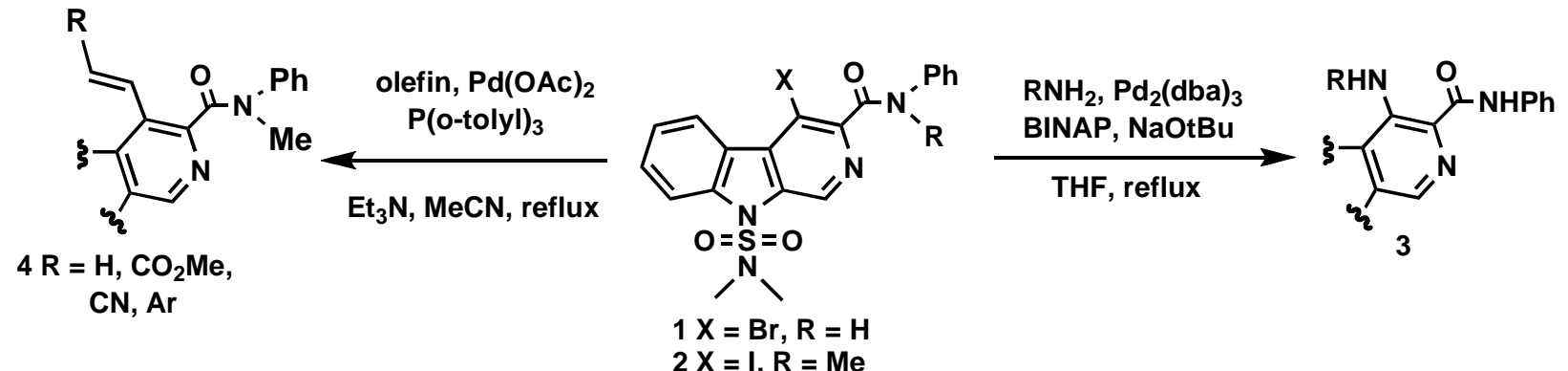

\section{Scheme 1}

\section{Results and Discussion}

The 4-iodo-3- $N$-methyl-3- $N$-phenyl- $\beta$-carboline-3-carboxamide 2 was prepared in a one-pot reaction starting from $3-N$-phenyl- $\beta$-carboline-3-carboxamide $\mathbf{5}$ (Scheme 2 ). ${ }^{7}$ Thus, the latter in THF was treated at $-78^{\circ} \mathrm{C}$ with 2.5 eq. of methyllithium and, after $45 \mathrm{~min}$, with iodine. At the end of the reaction period, excess methyl iodide was added. This provided compound $\mathbf{2}$ in 66\% yield together with a minor amount (16\%) of the non- $N$-methylated analogue of 2 . 


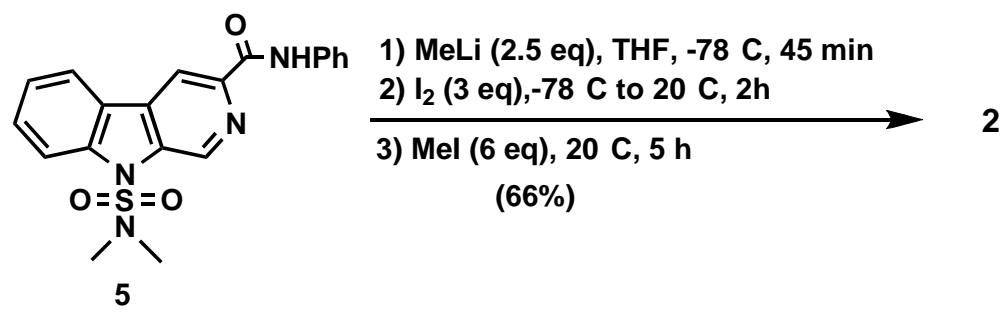

\section{Scheme 2}

Compound $\mathbf{2}$ was then used to study the reaction with arylboronic acids under SuzukiMiyaura palladium-catalyzed coupling conditions. ${ }^{11}$ Thus, treatment of $\mathbf{2}$ with phenylboronic acid $\mathbf{6 a}$ in the presence of $3 \mathrm{~mol}$.\% $\mathrm{Pd}\left(\mathrm{PPh}_{3}\right)_{4}$ and 2 eq $\mathrm{Na}_{2} \mathrm{CO}_{3}$ for $24 \mathrm{~h}$ in a mixture of ethanoltoluene at reflux provided the 4-phenyl derivative 7a in 90\% yield (Table 1). The 4-ethylphenyl$\beta$-carboline $\mathbf{7 b}$ was formed in slightly lower yield (85\%), while boronic acids with potentially sterically hindering ortho- substituents (2-methoxy, 2-fluoro, 2-formyl), on the phenyl ring also gave satisfactory yields of coupled product (7c, 7d, 7f, respectively). The presence of three methoxy groups on the phenyl ring (6e), however, completely inhibited the coupling reaction, and the formation of $\mathbf{7 e}$ was not observed. The introduction of heterocycles at C-4 using the same reaction conditions was also studied. Starting from 3-thienyl- and 3-furan- boronic acids, the coupled compounds $\mathbf{7 g}$ and $\mathbf{7 h}$ were obtained in good yields. However, in the case of pyridine-4-boronic acid, only traces of the expected coupled product $7 \mathbf{i}$ could be detected.

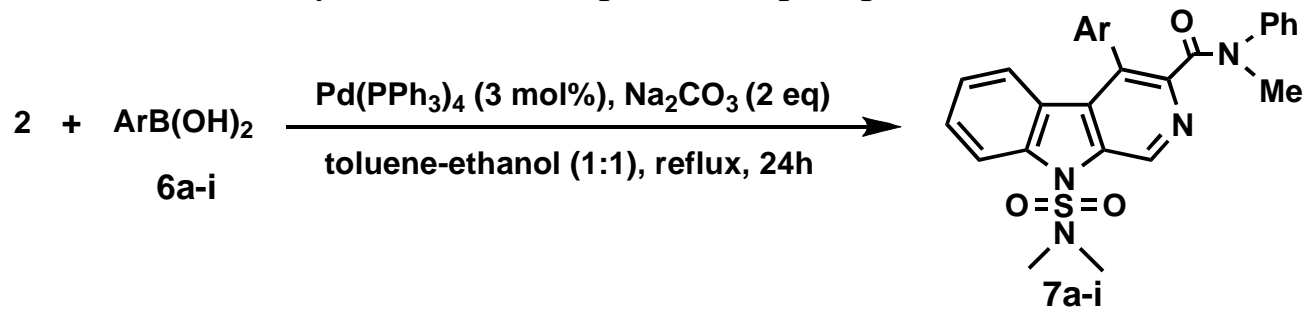

Table 1. Suzuki cross-coupling of arylboronic acids with compound $\mathbf{2}$

\begin{tabular}{|c|c|c|c|c|c|c|c|c|}
\hline $\begin{array}{l}\text { Cpd } \\
\text { No. }\end{array}$ & $\mathrm{Ar}$ & $\begin{array}{c}\text { Yield } \\
\% \\
\end{array}$ & $\begin{array}{l}\text { Cpd } \\
\text { No }\end{array}$ & $\mathrm{Ar}$ & $\begin{array}{c}\text { Yield } \\
\% \\
\end{array}$ & $\begin{array}{l}\text { Cpd } \\
\text { No }\end{array}$ & $\mathrm{Ar}$ & $\begin{array}{c}\text { Yield } \\
\% \\
\end{array}$ \\
\hline $7 a$ & & 90 & $7 d$ & & 82 & $7 \mathrm{~g}$ & & 78 \\
\hline $7 b$ & & 85 & $7 e$ & & 0 & $7 \mathrm{~h}$ & & 86 \\
\hline $7 c$ & & 73 & $7 f$ & & 90 & $7 \mathbf{i}$ & & trace \\
\hline
\end{tabular}


The 3,4,5-trimethoxyphenyl group is a common unit found in many cytotoxic compounds, particularly those known to interact with tubulin (e.g., colchicine, combretastatin, podophyllotoxin). For this reason, we decided to investigate reaction conditions which would allow preparation of the trimethoxyphenyl- $\beta$-carboline derivative $\mathbf{7 e}$ from $\mathbf{2}$, the classical Suzuki coupling conditions having failed. In particular, modifications of the base, solvents and palladium catalysts used in this reaction were made (Table 2). Thus, while replacement of sodium carbonate by potassium phosphate as the base had no beneficial effect (entries 1 and 2), use of potassium fluoride allowed isolation of compound $7 \mathbf{e}$ in $22 \%$ yield (entry 3). Use of DME-ethanol instead of toluene-ethanol as solvent system produced a small increase in yield of 7e (27\%, entry 4). While little improvement was observed when the reaction was conducted in the same solvent mixture but in the presence of cesium fluoride (entry 5), the yield of $\mathbf{7 e}$ increased to $38 \%$ when this latter base was employed in dioxane-methanol (entry 6). Finally, using conditions described by Broutin and Colobert ${ }^{12}$ for the efficient formation of $o$ - substituted biaryls (palladium diacetate in the presence of dppf as ligand, dioxane-ethanol, cesium fluoride and 4 h reflux), compound 7 e could be obtained in a very satisfactory $79 \%$ yield (entry 7). Application of these optimized conditions to the coupling of 4-iodo- $\beta$-carboline $\mathbf{3}$ with pyridine 4-boronic acid $\mathbf{6 i}$ did not, however, have any beneficial effect on the formation of compound $\mathbf{7 i}$.

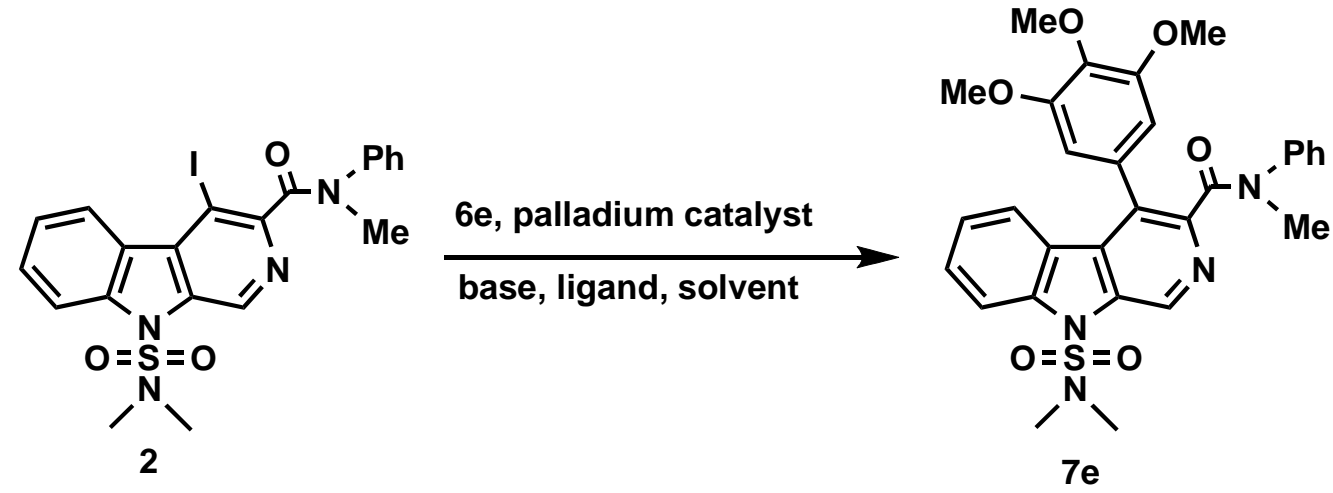

Table 2. Optimization of cross-coupling conditions of $\mathbf{6 e}$ with $\mathbf{2}$

\begin{tabular}{|c|c|c|c|c|c|}
\hline Entry & $\begin{array}{l}\text { Catalyst } \\
\text { (3 mol\%) }\end{array}$ & $\begin{array}{c}\text { Solvent } \\
(1: 1)\end{array}$ & $\begin{array}{l}\text { Base } \\
(3 \mathrm{eq})\end{array}$ & $\begin{array}{l}\text { Reflux } \\
\text { (h) }\end{array}$ & Yield of $7 e$ \\
\hline 1 & $\mathrm{Pd}\left(\mathrm{PPh}_{3}\right)_{4}$ & toluene: ethanol & $\mathrm{Na}_{2} \mathrm{CO}_{3}$ & 24 & 0 \\
\hline 2 & $\mathrm{Pd}\left(\mathrm{PPh}_{3}\right)_{4}$ & toluene: ethanol & $\mathrm{K}_{3} \mathrm{PO}_{4}$ & 24 & 0 \\
\hline 3 & $\mathrm{Pd}\left(\mathrm{PPh}_{3}\right)_{4}$ & toluene: ethanol & $\mathrm{KF}$ & 24 & 22 \\
\hline 4 & $\mathrm{Pd}\left(\mathrm{PPh}_{3}\right)_{4}$ & DME: ethanol & $\mathrm{KF}$ & 24 & 27 \\
\hline 5 & $\mathrm{Pd}\left(\mathrm{PPh}_{3}\right)_{4}$ & DME: ethanol & $\mathrm{CsF}$ & 24 & 28 \\
\hline 6 & $\mathrm{Pd}\left(\mathrm{PPh}_{3}\right)_{4}$ & dioxane:ethanol & $\mathrm{CsF}$ & 24 & 38 \\
\hline 7 & $\begin{array}{c}\mathrm{Pd}(\mathrm{OAc})_{2} \\
\mathrm{dppf}^{1}\end{array}$ & dioxane: ethanol & $\mathrm{CsF}^{2}$ & 4 & 79 \\
\hline
\end{tabular}

${ }^{1} 4.5$ mol. $\% ;^{2} 4$ equiv. 
Some of these substituted 4-aryl $\beta$-carbolines could be used for further transformations, as illustrated in Scheme 3. Thus, reaction of the $o$-formyl derivative $\mathbf{7 f}$ with benzylamine in acetic acid in the presence of sodium acetate, followed by reduction of the resulting imine with sodium borohydride in ethanol, provided the dibenzylamine 8. Treatment of the latter with sodium hydride in THF then provided, albeit in modest yield, the novel benzazepino- $\beta$-carboline $\mathbf{9}$.

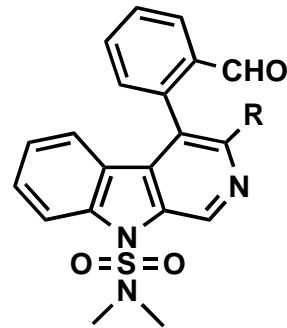

$7 f: R=C(=0) N(M e) P h$
1) $\mathrm{PhCH}_{2} \mathrm{NH}_{2}, \mathrm{NaOAC}$ $\mathrm{AcOH}$

2) $\mathrm{NaBH}_{4}, \mathrm{EtOH}, 0 \mathrm{C}$ (55\%)

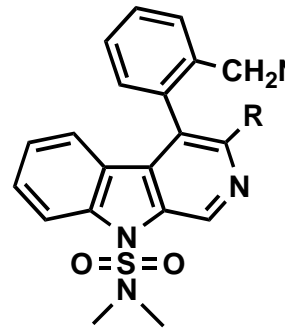

$8: \mathrm{R}=\mathrm{C}(=0) \mathrm{N}(\mathrm{Me}) \mathrm{Ph}$

\section{$\mathrm{CH}_{2} \mathrm{Ph}$}

$\mathrm{NaH}, \mathrm{TH}$

$0 \mathrm{C}, 5 \mathrm{~h}$

(20\%)

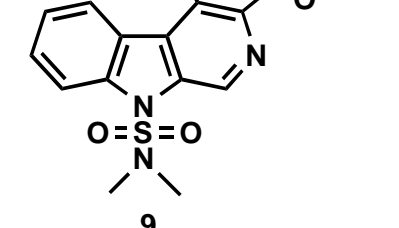

\section{Scheme 3}

As we have previously demonstrated, the $N, N$-dimethylsulfamoyl protecting group of the $N-9$ position of $\beta$-carbolines can be efficiently cleaved using samarium iodide or trifluoromethanesulfonic acid $^{7}$ as well as electrochemically. ${ }^{13}$ In the case of the 4-aryl $\beta$ carbolines, treatment of $\mathbf{7 a - e}$ with a 1:10:1 mixture of trifluoromethanesulfonic acid, trifluoroacetic acid and anisole at room temperature for $1 \mathrm{~h}$ provided the corresponding $N-9$ deprotected derivatives 10a-e in yields of 84 to 92\% (Scheme 4).
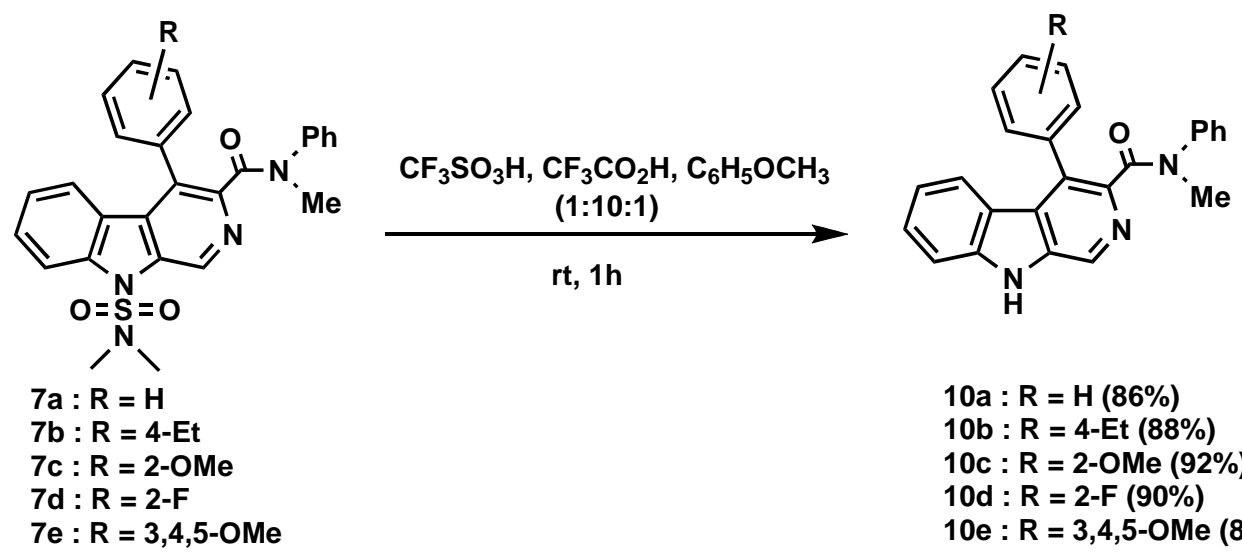

$7 \mathrm{a}: \mathrm{R}=\mathrm{H}$

$7 b: R=4-E t$

$7 \mathrm{e}: \mathrm{R}=3,4,5-\mathrm{OMe}$
$10 \mathrm{a}: \mathrm{R}=\mathrm{H}(\mathbf{8 6 \% )}$

$10 \mathrm{~b}: \mathrm{R}=4-\mathrm{Et}(\mathbf{8 8 \% )}$

$10 \mathrm{c}: \mathrm{R}=2-\mathrm{OMe}(92 \%)$

$10 \mathrm{~d}: \mathrm{R}=2-\mathrm{F}(90 \%)$

$10 \mathrm{e}: \mathrm{R}=3,4,5-\mathrm{OMe}(84 \%)$

\section{Scheme 4}

In conclusion, the $N$-9-protected 4-iodo- $N$-methyl- $N$-phenyl- $\beta$-carboline-3-carboxamide $\mathbf{2}$, obtained by ortho- directed metalation of $\beta$-carboline-3-carboxamide $\mathbf{5}$, undergoes efficient Suzuki cross-coupling reactions with a variety of aryl- and heteroaryl-boronic acids to give the corresponding 4-aryl- $\beta$-carboline-3-carboxamides. While these cross-coupling reactions were 
generally successful using the classical Suzuki-Miyaura conditions, the reaction parameters required optimization in order to prepare the 3,4,5-trimethoxyphenyl derivative 7e. These compounds can be conveniently deprotected to afford novel $\beta$-carboline derivatives (10a-e) suitable for pharmacological evaluation, notably with respect to the $\mathrm{GABA}_{\mathrm{A}}$ receptor. Finally, the incorporation of functional groups on the C-4 phenyl ring can allow further transformations to potentially active $\beta$-carboline derivatives as shown by the preparation of the benzazepino- $\beta$ carboline 9 from the 4-(2-formylphenyl) precursor $\mathbf{7 f}$.

\section{Experimental Section}

General Procedures. Melting points were measured in capillary tubes on a Büchi B-540 apparatus and are uncorrected. IR spectra were recorded with a Perkin Elmer Spectrum BX FTIR spectrometer. ${ }^{1} \mathrm{H}$ NMR and ${ }^{13} \mathrm{C}$ NMR spectra were measured respectively at $300 \mathrm{MHz}, 250$ $\mathrm{MHz}$ or $75 \mathrm{MHz}$ on a Bruker Aspect 3000 (300 MHz) instrument. RT denotes room temperature. Chemical shifts are given as $\delta$ values with reference to $\mathrm{Me}_{4} \mathrm{Si}$ as internal standard. Electronimpact- and chemical- ionization- mass spectra were recorded on an Automass Multi Thermo Finnigan and AEI MS-9 spectrometer, respectively. High-resolution mass spectra were obtained using an ESI-TOF Micromass LCT spectrometer. TLC and preparative chromatography were performed on Merck silica gel 60 plates with fluorescent indicator. The plates were visualized with UV light (254 and $366 \mathrm{~nm}$ ) or with a 3.5\% solution of phosphomolybdic acid in ethanol. All column chromatography was conducted on Merck 60 silica gel (230-400 mesh) at medium pressure (200 mbar). All solvents were distilled before use. Reagents and chemicals were purchased from the Aldrich Chemical Co. and were used without further purification. Elemental analyses were performed at the ICSN, CNRS, Gif-sur-Yvette.

9-( $N, N$-Dimethylsulfamoyl)-4-iodo-3- $N$-methyl-3- $N$-phenyl- $\beta$-carboline-3-carboxamide (2). A solution of compound $\mathbf{5}^{7}(1.0 \mathrm{~g}, 2.54 \mathrm{mmol})$ in anhydrous THF was treated dropwise at $-78^{\circ} \mathrm{C}$ under argon with a solution of methyllithium in THF (5.6 mL of a $1.0 \mathrm{M}$ solution; $5.6 \mathrm{mmol}$ ). The solution was stirred for $45 \mathrm{~min}$ and a solution of iodine (1.6 g, $6.3 \mathrm{mmol})$ in THF (10 mL) was added dropwise. The reaction mixture was stirred for a further $30 \mathrm{~min}$ at $-78^{\circ} \mathrm{C}$ and then for $2 \mathrm{~h}$ at RT. Freshly distilled methyl iodide $(0.80 \mathrm{~mL}, 12.7 \mathrm{mmol})$ was then added and stirring was maintained at RT for $5 \mathrm{~h}$. Ice-cold water $(30 \mathrm{~mL})$ was added and the volume was reduced to three-quarters by evaporation under reduced pressure. Ethyl acetate (75 mL) was added, the mixture was washed with saturated aqueous $\mathrm{Na}_{2} \mathrm{~S}_{2} \mathrm{O}_{3}(3 \times 10 \mathrm{~mL})$ and then with saturated aqueous $\mathrm{NaCl}(25 \mathrm{~mL})$. The organic phase was dried over $\mathrm{MgSO}_{4}$, the solvents were removed under vacuum and the residue was purified by column chromatography on silica gel (ethyl acetate-heptane 1: 2). The non- $N$-methylated 4-iodo analogue of 2 (210 mg, 16\%) was eluted first, followed by the $N$-methyl-4-iodo compound 2 (R = Me; $880 \mathrm{mg}, 65 \%$ ), obtained as a white solid, mp $237^{\circ} \mathrm{C}:{ }^{1} \mathrm{H}$ NMR (DMSO-d $\left.6300 \mathrm{MHz}\right) \delta 2.74$ (s, 6H), 3.59 (s, 3H), 7.00 (t, 1H, J = 
$8.0 \mathrm{~Hz}$ ), 7.08 (d, 2H, J = 7.6 Hz), 7.30 (d, 2H, J = 7.6 Hz), 7.46 (dd, 1H, J = 8.0 and $7.7 \mathrm{~Hz}$ ), 7.65 (dd, $1 \mathrm{H}, \mathrm{J}=7.7$ and $8.5 \mathrm{~Hz}$ ), 8.18 (d, 1H, J = $8.5 \mathrm{~Hz}$ ), 9.04 (d, 1H, J = $8.0 \mathrm{~Hz}$ ), $9.25(\mathrm{~s}, 1 \mathrm{H}) ;{ }^{13} \mathrm{C}$ NMR (DMSO-d 6 , 75 MHz) $\delta$ 37.2, 38.7, 114.7, 122.5, 122.9, 124.0, 125.9, 127.0, 127.1, 128.8, 129.3, 130.6, 130.9, 134.6, 135.0, 136.0, 142.8, 168.9; CIMS m/z 535 (MH+); IR (film): 1646, $1171 \mathrm{~cm}^{-1}$. Anal. Calcd for $\mathrm{C}_{21} \mathrm{H}_{19} \mathrm{~N}_{4} \mathrm{O}_{3} \mathrm{Si}$ : C, 47.20; H, 3.58; N, 10.48; I, 23.75; S, 6,00. Found: C, 47.64; H, 3.56; N, 10.35; I, 23.45; S, 5.85\%.

\section{General procedure for the preparation of 4-aryl- $\beta$-carboline-3-carboxamides 7}

To a solution of compound 2 (54 mg, $0.1 \mathrm{mmol}$ ) in toluene-ethanol (12 mL of a 1:1 mixture) were added aqueous sodium carbonate ( $3 \mathrm{~mL}$ of a $1.5 \mathrm{M}$ solution; $4.5 \mathrm{mmol}$ ), the arylboronic acid (0.2 mmol) and palladium tetrakis(triphenylphosphine) (0.003 mmol, $3 \mathrm{~mol} \%$ ). The mixture was degassed with argon for $5 \mathrm{~min}$ and heated at reflux for $24 \mathrm{~h}$. The reaction mixture was then cooled, diluted with ethyl acetate $(15 \mathrm{~mL})$ and washed with water $(20 \mathrm{~mL})$. The organic phase was dried over $\mathrm{MgSO}_{4}$ and the solvents were removed under reduced pressure leaving the crude product which was purified by column chromatography on silica gel (dichloromethane-ethyl acetate, 3:1).

9-( $N, N$-Dimethylsulfamoyl)-3- $N$-methyl-3- $N$-phenyl-4-phenyl- $\beta$-carboline-3-carboxamide

(7a). This compound, prepared from phenylboronic acid using the above general procedure, was isolated as a white solid in $98 \%$ yield, mp $98^{\circ} \mathrm{C}:{ }^{1} \mathrm{H}$ NMR $\left(\mathrm{CD}_{3} \mathrm{CN}, 400 \mathrm{MHz}\right): \delta 2.70(\mathrm{~s}, 6 \mathrm{H})$, 3.30 (s, 3H), 7.10 (m, 1H), 7.25 (m, 1H), 7.31-7.40 (m, 3H), 7.42 (t, 1H, J = 8.0 Hz), 7.55 (m, 4H), 7.88 (t, 2H), 8.10 (d, 1H, J = $8.6 \mathrm{~Hz}), 8.55$ (d, 2H, J = $8.0 \mathrm{~Hz}), 9.10(\mathrm{~s}, 1 \mathrm{H}) ;{ }^{13} \mathrm{C} \mathrm{NMR}$ $\left(\mathrm{CD}_{3} \mathrm{CN}, 75 \mathrm{MHz}\right): \delta$ 36.5, 38.8 (2C), 114.2, 117.8, 120.2, 121.5, 124.7, 124.9, 127.4, 127.5 (4C), 128.0, 128.6, 129.1 (4C), 129.9, 132.2, 135.1, 135.4, 139.0, 150.4, 160.1; CIMS m/z 485 $[\mathrm{M}+\mathrm{H}]^{+}$; IR (film): 1616, 1592, $1173 \mathrm{~cm}^{-1}$; HRCIMS m/z calcd for $\mathrm{C}_{27} \mathrm{H}_{24} \mathrm{SN}_{4} \mathrm{O}_{3}+\mathrm{Na}^{+}(\mathrm{M}+$ $\mathrm{Na}^{+}$) 507.1467, found 507.1459.

9-( $N, N$-Dimethylsulfamoyl)-3- $N$-methyl-3- $N$-phenyl-4-(4-ethylphenyl)- $\beta$-carboline-3-

carboxamide (7b). This compound, prepared from 4-ethylphenylboronic acid using the above general procedure, was obtained as a white solid in $85 \%$ yield, mp $79-81^{\circ} \mathrm{C}:{ }^{1} \mathrm{H} \mathrm{NMR}\left(\mathrm{CD}_{3} \mathrm{CN}\right.$, $400 \mathrm{MHz}) \delta 1.5$ (t, 3H), 2.8 (s, 6H), 3.3 (s, 3H), 4.8 (q, 2H), 6.6 (dd, 2H, J = 8.3 Hz), 6.9-7.1 (m, 2H), $7.4(\mathrm{~m}, 1 \mathrm{H}), 7.5(\mathrm{~m}, 4 \mathrm{H}), 7.5-7.6(\mathrm{~m}, 2 \mathrm{H}), 7.48(\mathrm{~d}, 1 \mathrm{H}), 8.0(\mathrm{~d}, 1 \mathrm{H}, \mathrm{J}=8.6 \mathrm{~Hz}), 9.2(\mathrm{~s}, 1 \mathrm{H})$; ${ }^{13} \mathrm{C}$ NMR $\left(\mathrm{CD}_{3} \mathrm{CN}, 75 \mathrm{MHz}\right) \delta$ 14.8, 27.8 (2C), 35.4, 37.7, 114.7, 122.8 (2C), 125.6, 126.0, 127.4, 127.8 (2C), 128.0 (2C), 128.8 (2C), 129.1, 129.3, 131.0, 133.6, 134.2, 134.2, 139.8, 140.0, 142.0, 144.5, 147.2, 167.3; IR (film): 1649, 1166; HRCIMS m/z calcd for $\mathrm{C}_{29} \mathrm{H}_{28} \mathrm{~N}_{4} \mathrm{O}_{3} \mathrm{~S}+\mathrm{H}^{+}$ $\left(\mathrm{MH}^{+}\right)$513.1938, found 513.1960.

9-( $N, N$-Dimethylsulfamoyl)-3- $N$-methyl-3- $N$-phenyl-4-(2-methoxyphenyl)- $\beta$-carboline-3carboxamide (7c). This compound, prepared from 2-methoxyphenylboronic acid using the above general procedure, was obtained as a white solid in $73 \%$ yield, mp $74-75^{\circ} \mathrm{C}$ : ${ }^{1} \mathrm{H} \mathrm{NMR}$ $\left(\mathrm{CD}_{3} \mathrm{CN}, 400 \mathrm{MHz}\right) \delta 2.8$ (s, 6H), 3.3 (s, 3H), 3.5 (s, 3H), 6.67 (d, 1H, J = $8.2 \mathrm{~Hz}$ ), 6.87 (d, 1H, J $=7.8 \mathrm{~Hz}), 7.0(\mathrm{~m}, 2 \mathrm{H}), 7.15(\mathrm{~m}, 3 \mathrm{H}), 7.4(\mathrm{~m}, 2 \mathrm{H}), 7.5-7.6(\mathrm{~m}, 3 \mathrm{H}), 8.03$ (d, 1H, J = $8.5 \mathrm{~Hz}), 9,2$ (s, 1H); ${ }^{13} \mathrm{C}$ NMR $\left(\mathrm{CD}_{3} \mathrm{CN}, 75 \mathrm{MHz}\right) \delta$ 37.2, 38.9 (2C), 55.3, 111.9, 115.8 (2C), 121.6, 121.8 
(2C), 124.5, 127.1, 127.7, 127.8, 129.6 (2C), 129.9, 130.1 (2C), 130.9, 133.1, 133.7, 133.8, 135.6, 143.5, 158.6, 166.3, 170.5; IR (film): 1649, 1604, $1153 \mathrm{~cm}^{-1}$; HRCIMS $\mathrm{m} / \mathrm{z}$ calcd for $\mathrm{C}_{28} \mathrm{H}_{26} \mathrm{~N}_{4} \mathrm{O}_{4} \mathrm{~S}+\mathrm{Na}^{+}\left(\mathrm{M}+\mathrm{Na}^{+}\right)$537.1572, found 537.1550.

9-(,$N$-Dimethylsulfamoyl)-3- $N$-methyl-3- $N$-phenyl-4-(2-fluorophenyl)- $\beta$-carboline-3-

carboxamide (7d). This compound, prepared from 2-fluorophenylboronic acid using the above general procedure, was obtained as a white solid in $82 \%$ yield, mp $210-211^{\circ} \mathrm{C}:{ }^{1} \mathrm{H}$ NMR $\left(\mathrm{CD}_{3} \mathrm{CN}, 400 \mathrm{MHz}\right) \delta 2.7(\mathrm{~s}, 6 \mathrm{H}), 3.2(\mathrm{~s}, 3 \mathrm{H}), 6.7(\mathrm{~d}, 1 \mathrm{H}, \mathrm{J}=6.7 \mathrm{~Hz}), 6.9(\mathrm{~d}, 1 \mathrm{H}, \mathrm{J}=6.7 \mathrm{~Hz})$, $7.2(\mathrm{t}, 2 \mathrm{H}, \mathrm{J}=8.9 \mathrm{~Hz}), 7.3(\mathrm{~m}, 5 \mathrm{H}), 7.5(\mathrm{t}, 1 \mathrm{H}, \mathrm{J}=8.4 \mathrm{~Hz}), 7.6$ (d, 1H, J = $8.6 \mathrm{~Hz}), 8.0$ (d, 1H, J = $8.4 \mathrm{~Hz}$ ), 9.2 (s, $1 \mathrm{H}) ;{ }^{13} \mathrm{C}$ NMR $\left(\mathrm{CD}_{3} \mathrm{CN}, 75 \mathrm{MHz}\right) \delta 38.9$, 39.0 (2C), 116.0, 116.1, 117.1, 117.3, 123.5, 123.7, 124.2, 124.8, 125.2, 127.8 (2C), 128.1, 129.8, 129.9, 128.2, 130.0, 130.2, 131.3, 131.6, 132.9, 136.2, 136.4, 143.5, 148.6-170.1 (C-F), 168.6; IR (film): 1649, $1168 \mathrm{~cm}^{-1}$; HRCIMS $\mathrm{m} / \mathrm{z}$ calcd for $\mathrm{C}_{27} \mathrm{H}_{23} \mathrm{~N}_{4} \mathrm{O}_{3} \mathrm{SF}+\mathrm{Na}^{+}\left(\mathrm{M}+\mathrm{Na}^{+}\right)$525.1373, found 525.1383.

9-( $N, N$-Dimethylsulfamoyl)-3- $N$-methyl-3- $N$-phenyl-4-(2-formylphenyl)- $\beta$-carboline-3-

carboxamide (7f). This compound, prepared from 2-formylphenylboronic acid using the above general procedure, was obtained as a white solid in $90 \%$ yield, mp $99-101^{\circ} \mathrm{C}:{ }^{1} \mathrm{H} N M R\left(\mathrm{CD}_{3} \mathrm{CN}\right.$, $400 \mathrm{MHz}) \delta 2.90$ (s, 6H), 3.10 (s, 3H), $6.60(\mathrm{~m}, 1 \mathrm{H}), 7.00(\mathrm{~m}, 1 \mathrm{H}), 7.1-7.3(\mathrm{~m}, 4 \mathrm{H}), 7.40(\mathrm{t}, 1 \mathrm{H}$, $\mathrm{J}=8.0 \mathrm{~Hz}$ ), 7.55 (t, 1H, J = 8.6), $7.8(\mathrm{t}, 1 \mathrm{H}), 7.9(\mathrm{t}, 1 \mathrm{H}), 8.10$ (d, 1H, J = $8.6 \mathrm{~Hz}), 8.2$ (d, 2H, J = $8.0 \mathrm{~Hz}$ ), 9.10 (s, $1 \mathrm{H}), 9.30(\mathrm{~s}, 1 \mathrm{H}) ;{ }^{13} \mathrm{C} \mathrm{NMR}\left(\mathrm{CD}_{3} \mathrm{CN}, 75 \mathrm{MHz}\right) \delta 35.7,37.8(2 \mathrm{C}), 114.5,117.0$, 120.1, 121.6, 122.2, 123.2, 124.0, 124.4, 125.6, 126.1, 127.0 (2C), 128.2, 128.4 (2C), 129.5, 130.5, 133.9, 134.5, 134.8, 139.6, 142.4, 146.9, 167.4, 190.1; IR (film): 3435, 1697, 1651, 1173 $\mathrm{cm}^{-1}$; HRCIMS $\mathrm{m} / \mathrm{z}$ calcd for $\mathrm{C}_{28} \mathrm{H}_{24} \mathrm{SN}_{4} \mathrm{O}_{4}+\mathrm{Na}^{+}\left(\mathrm{M}+\mathrm{Na}^{+}\right)$535.1416, found 535.1447.

9-( $N, N$-Dimethylsulfamoyl)-3- $N$-methyl-3- $N$-phenyl-4-(3-furanyl)- $\beta$-carboline-3-

carboxamide (7g). This compound, prepared from 3-furanboronic acid using the above general procedure, was obtained as a white solid in $78 \%$ yield, mp 96-98 ${ }^{\circ} \mathrm{C}:{ }^{1} \mathrm{H}$ NMR $\left(\mathrm{CD}_{3} \mathrm{OD}, 400\right.$ MHz) $\delta 2.7$ (s, 6H); 3.2 (s, 3H), 6.30 (s, 1H), 6.80 (d, 1H, J = $7.58 \mathrm{~Hz}$ ), 6.98 (d, J = 7.7 Hz), 7.0 (m, 4H), 7.30 (t, 1H, J = $7.7 \mathrm{~Hz}$ ), 7.4 (t, 1H, J = 8.4 Hz), 7.5 (d, 1H, J = $7.8 \mathrm{~Hz}$ ), 7.7 (s, 1H), 8.0 (d, $1 \mathrm{H}, \mathrm{J}=8.4 \mathrm{~Hz}$ ), $9.18(\mathrm{~s}, 1 \mathrm{H}) ;{ }^{13} \mathrm{C}$ NMR $\left(\mathrm{CD}_{3} \mathrm{OD}, 75 \mathrm{MHz}\right) \delta 38.9,39.0$ (2C), 56.0, 103.0, 113.4, 115.9, 116.3, 120.2, 122.9, 125.1, 127.1, 128.1 (2C), 128.3, 129.7 (2C), 133.1, 135.7, 136.5, 141.3, 143.5, 145.1, 149.0, 170.6; IR (film): 1649, $1171 \mathrm{~cm}^{-1}$; HRCIMS $\mathrm{m} / \mathrm{z}$ calcd for $\mathrm{C}_{25} \mathrm{H}_{22} \mathrm{~N}_{4} \mathrm{O}_{4} \mathrm{~S}+\mathrm{Na}^{+}\left(\mathrm{M}+\mathrm{Na}^{+}\right)$497.1259, found 497.1273.

\section{9-( $N, N$-Dimethylsulfamoyl)-3- $N$-methyl-3- $N$-phenyl-4-(3-thienyl)- $\beta$-carboline-3-}

carboxamide (7h). This compound, prepared from 3-thienylboronic acid using the above general procedure, was obtained as a white solid in $86 \%$ yield, mp $96-98^{\circ} \mathrm{C}:{ }^{1} \mathrm{H}$ NMR $\left(\mathrm{CD}_{3} \mathrm{CN}\right.$, $400 \mathrm{MHz}$ ) $\delta 2.7$ (s, 6H), 3.53 (s, 3H), 7.05 (d, 1H, J = $7.58 \mathrm{~Hz}$ ), 7.15 (m, 5H), 7.20 (s, 1H), 7.29 (d, 1H, J = 7.4 Hz), 7.56 (t, 1H, J = 7.4 Hz), 7.76 (t, 1H, J =7.3 Hz), 8.21 (d, 1H, J = 8.4 Hz), $9.06\left(\mathrm{~d}, 1 \mathrm{H}, \mathrm{J}=8.0 \mathrm{~Hz}\right.$ ), $9.23(\mathrm{~s}, 1 \mathrm{H})$; IR (film): 1647, $1171 \mathrm{~cm}^{-1}$; HRCIMS $\mathrm{m} / \mathrm{z}$ calcd for $\mathrm{C}_{25} \mathrm{H}_{22} \mathrm{~N}_{4} \mathrm{O}_{3} \mathrm{~S}_{2}+\mathrm{Na}^{+}\left(\mathrm{M}+\mathrm{Na}^{+}\right)$513.1031, found 513.1052.

9-( $N, N$-Dimethylsulfamoyl)-3- $N$-methyl-3- $N$-phenyl-4-(3,4,5-trimethoxyphenyl)- $\beta$ -

carboline-3-carboxamide (7e). To a solution of 3,4,5-trimethoxyphenylboronic acid (40 mg, $0.20 \mathrm{mmol})$ in a $1: 1$ mixture of dioxane and ethanol $(4 \mathrm{~mL})$ was added cesium fluoride $(58 \mathrm{mg}$, 
$0.40 \mathrm{mmol}$ ) followed, after $10 \mathrm{~min}$ of stirring, by $\mathrm{Pd}(\mathrm{OAc})_{2}(0.7 \mathrm{mg}, 0.003 \mathrm{mmol}, 3 \mathrm{~mol}$ \%), dppf (2.5 mg, $0.0045 \mathrm{mmol}, 4.5 \mathrm{~mol}$. \%) and compound 2 (54 mg, $0.1 \mathrm{mmol}$ ). The mixture was refluxed for $4 \mathrm{~h}$, the solvents were removed under vacuum, the residue was taken up in dichloromethane $(10 \mathrm{~mL})$ and the solution was washed with water $(10 \mathrm{~mL})$. The organic phase was dried over $\mathrm{MgSO}_{4}$, the solvent was removed under vacuum and the crude product was purified by column chromatography on silica gel (dichloromethane-ethyl acetate 1:1) affording compound 7e (45 mg, 79\%) as a white solid, mp $178-180^{\circ} \mathrm{C}:{ }^{1} \mathrm{H} \mathrm{NMR}\left(\mathrm{CD}_{3} \mathrm{CN}, 400 \mathrm{MHz}\right) \delta 2.8$ (s, 6H), 3.3 (s, 3H), 3.8 (s, 6H), 3.9 (s, 3H), 6.42 (s, 2H), 6.75 (d, 1H, J = 7.58 Hz), 7.10 (t, 2H, J $=7.5 \mathrm{~Hz}), 7.29$ (t, 1H, J = 7.4 Hz), $7.56(\mathrm{t}, 1 \mathrm{H}, \mathrm{J}=7.4 \mathrm{~Hz}), 7.6(\mathrm{t}, 1 \mathrm{H}, \mathrm{J}=7.3 \mathrm{~Hz}), 7.7$ (d, 1H, J = $8.4 \mathrm{~Hz}), 8.18(\mathrm{~d}, 1 \mathrm{H}, \mathrm{J}=8.0 \mathrm{~Hz}), 9.38(\mathrm{~s}, 1 \mathrm{H}) ;{ }^{13} \mathrm{C} \mathrm{NMR}\left(\mathrm{CD}_{3} \mathrm{CN}, 75 \mathrm{MHz}\right) \delta 35.4,37.8(2 \mathrm{C})$, 55.2 (2C), 59.3, 106.8 (2C), 114.4, 122.9, 123.3, 125.7, 126.0, 127.6 (2C), 127.9, 128.3, 128.5, 128.6 (2C), 129.3, 131.4, 131.7, 134.3, 139.0, 140.1, 152.9, 153.9 (2C), 168.2; IR (film): 1645, 1581, $1173 \mathrm{~cm}^{-1}$; HRCIMS $\mathrm{m} / \mathrm{z}$ calcd for $\mathrm{C}_{30} \mathrm{H}_{30} \mathrm{~N}_{4} \mathrm{O}_{6} \mathrm{~S}+\mathrm{Na}^{+}\left(\mathrm{M}+\mathrm{Na}^{+}\right)$597.1784, found 597.1803.

\section{2- $N$-Benzyl-6-( $N, N$-dimethylsulfamoyl)-1,4-dihydrobenzazepin-3-oxo-[4,5-c]- $\beta$-carboline}

(9). A solution of compound $7 \mathbf{d}(10 \mathrm{mg}, 0.02 \mathrm{mmol})$ in ethanol $(15 \mathrm{~mL})$ was cooled to $0^{\circ} \mathrm{C}$ and benzylamine $(0.6 \mathrm{mg}, 0.0056 \mathrm{mmol})$ followed by a buffer solution $(\mathrm{pH} 7)$ composed of sodium acetate $(1.1 \mathrm{mg})$ in acetic acid $(5 \mathrm{~mL})$ were added. After $1 \mathrm{~h}$ of stirring, sodium borohydride (7.8 $\mathrm{mg}, 0.2 \mathrm{mmol}$ ) was slowly added to the mixture and stirring was continued for $3 \mathrm{~h}$ at $0^{\circ} \mathrm{C}$. The solution was diluted with diethyl ether $(15 \mathrm{~mL})$, washed with water $(3 \times 5 \mathrm{~mL})$, dried over $\mathrm{MgSO}_{4}$ and the solvents removed under reduced pressure. The residue was dissolved in anhydrous THF $(15 \mathrm{~mL})$ and cooled to $0^{\circ} \mathrm{C}$ under argon. Sodium hydride $(1.2 \mathrm{mg}$ of a $50 \%$ suspension in oil, $0.025 \mathrm{mmol}$ ) was added and after $5 \mathrm{~h}$ of stirring at $0^{\circ} \mathrm{C}$, water $(5 \mathrm{~mL})$ was added. The mixture was concentrated to half volume under vacuum, ethyl acetate $(15 \mathrm{~mL})$ was added and the solution was washed with water $(3 \times 10 \mathrm{~mL})$. The organic phase was dried over magnesium sulfate, the solvent was removed under reduced pressure and the crude product was purified by preparative tlc (toluene-acetone 2:1) affording compound 9 (1.9 mg, $0.004 \mathrm{mmol}, 20 \%$ ) as a white solid, mp 245-246 ${ }^{\circ} \mathrm{C}:{ }^{1} \mathrm{H}$ NMR (DMSO-d 6 , $\left.300 \mathrm{MHz}\right) \delta 2.70(\mathrm{~s}, 6 \mathrm{H}), 4.0(\mathrm{~d}, 1 \mathrm{H}, \mathrm{J}=14.6$ $\mathrm{Hz}$ ), 4.15 (d, 1H, J = 14.6 Hz), 4.20 (d, 1H, J = 6.2 Hz), 4.80 (d, 1H, J = 6.2 Hz), 7.0-7.1 (m, 1H), 7.2-7.3 (m, 5H), 7.4-7.5 (m, 5H), 7.7 (d, J = 8.2 Hz), 8.0 (d, 1H, J = 8.5 Hz), 9.25 (s, 1H); ${ }^{13} \mathrm{C}$ NMR (75 MHz, DMSO-d $\left.{ }_{6}\right) \delta 39.4,39.7$ (2C), 49.4, 114.6, 122.5, 122.9, 123.1, 127.1, 127.2, 127.4, 127.6, 127.7, 127.8, 128.4 (2C), 129.5 (2C), 129.9, 131.2, 132.0, 135.6, 135.9, 137.5, 139.3, 139.5, 145.4, 165.5; EIMS m/z $496\left(\mathrm{M}^{+}\right)$; IR (film): 1647, $1174 \mathrm{~cm}^{-1}$. Anal. Calcd for $\mathrm{C}_{28} \mathrm{H}_{24} \mathrm{~N}_{4} \mathrm{O}_{3} \mathrm{~S} .0 .25\left(\mathrm{CH}_{3}\right)_{2} \mathrm{CO}$ : C, 65.75; H, 4.70; N, 10.95; S, 6.26. Found: C, 65.65; H, 5.01; N, 10.75; S, 6.16\%.

\section{General procedure for deprotection of the 9-( $N, N$-dimethylsulfamoyl)-4-aryl- $\beta$-carboline- 3-carboxamides}

A solution of the $\beta$-carboline (7a-7e, $0.07-0.08 \mathrm{mmol})$ in a 1:1:10 mixture of anisole, trifluoromethanesulfonic acid and trifluoroacetic acid $(1.5 \mathrm{~mL})$ was stirred at RT for $1 \mathrm{~h}$. The 
reaction mixture was concentrated under vacuum, ice-cold water $(5 \mathrm{~mL})$ was added to the residue and the mixture was made basic with $4 N$ aqueous sodium hydroxide solution. The precipitate was collected by filtration and washed successively with water and with ethyl acetate. The solid was suspended in ethanol and the mixture was filtered once again to afford the $N-9$ deprotected compounds 10a-10e.

$N$-Methyl- $N$-phenyl-4-phenyl- $\beta$-carboline-3-carboxamide (10a). This compound, prepared from $7 \mathrm{a}$ (40 $\mathrm{mg}, 0.08 \mathrm{mmol}$ ) using the above general procedure, was obtained as a yellow powder (27 mg, $0.07 \mathrm{mmol}, 86 \%$ ), mp 149-150 ${ }^{\circ} \mathrm{C}:{ }^{1} \mathrm{H}$ NMR (DMSO-d 6 , $300 \mathrm{MHz}$ ) $\delta 3.27$ (s, 3H), $7.10(\mathrm{~m}, 1 \mathrm{H}), 7.20(\mathrm{~m}, 1 \mathrm{H}), 7.30-7.40$ (m, 3H), 7.40 (t, 1H, J = $8.0 \mathrm{~Hz}), 7.75$ (m, 4H), 7.9 (t, 2H), $8.0(\mathrm{~d}, 1 \mathrm{H}, \mathrm{J}=8.6 \mathrm{~Hz}), 8.60(\mathrm{~d}, 2 \mathrm{H}, \mathrm{J}=8.0 \mathrm{~Hz}), 9.10(\mathrm{~s}, 1 \mathrm{H}), 11.9(\mathrm{~s}, 1 \mathrm{H}) ;{ }^{13} \mathrm{C}$ NMR (DMSO-d 6 , 75 MHz) $\delta 36.8,111.8,114.2$ (2C), 117.9, 120.0, 121.3, 121.9, 122.2, 123.5, 124.7 (2C), 125.1, 127.9, 129.0 (4C), 130.3, 132.7 (2C), 135.9, 139.3, 150.8, 160.0; IR (film): 3202, 1619, $1592 \mathrm{~cm}^{-1}$; HRCIMS $\mathrm{m} / \mathrm{z}$ calcd for $\mathrm{C}_{25} \mathrm{H}_{19} \mathrm{~N}_{3} \mathrm{O}+\mathrm{Na}^{+}\left(\mathrm{M}+\mathrm{Na}^{+}\right)$400.1426, found 400.1427.

$N$-Methyl- $N$-phenyl-4-(4-ethylphenyl)- $\beta$-carboline-3-carboxamide (10b). This compound, prepared from $7 \mathbf{b}$ (35 $\mathrm{mg}, 0.07 \mathrm{mmol}$ ) using the above general procedure, was obtained as an off-white solid (24 mg, $0.06 \mathrm{mmol}, 88 \%$ ), $\mathrm{mp}>300^{\circ} \mathrm{C}:{ }^{1} \mathrm{H}$ NMR (DMSO- $\mathrm{d}_{6}, 300 \mathrm{MHz}$ ) $\delta 1.30$ (t, 3H), $3.7(\mathrm{~s}, 3 \mathrm{H}), 4.0(\mathrm{~m}, 2 \mathrm{H}), 6.85-7.0(\mathrm{~m}, 5 \mathrm{H}), 7.09(\mathrm{~m}, 3 \mathrm{H}), 7.50(\mathrm{~d}, 2 \mathrm{H}, \mathrm{J}=8.6 \mathrm{~Hz}), 7.5-$ $7.6(\mathrm{~m}, 1 \mathrm{H}), 7.68(\mathrm{~d}, 1 \mathrm{H}, \mathrm{J}=7.6 \mathrm{~Hz}), 8.15(\mathrm{~d}, 1 \mathrm{H}, \mathrm{J}=8.2 \mathrm{~Hz}), 8.9(\mathrm{~s}, 1 \mathrm{H}), 12.0(\mathrm{~s}, 1 \mathrm{H}) ;{ }^{13} \mathrm{C}$ NMR (DMSO-d 6 , $75 \mathrm{MHz}) \delta 16.1,28.6,37.9,112.7,114.0,114.9,119.8,120.8,123.1(2 \mathrm{C})$, 126.2, 128.1 (2C), 128.8, 129.7 (2C), 129.9 (2C), 130.2, 131.0, 132.1, 135.8, 141.8, 143.0, 144.7, 158.8, 168.5; IR (film): 3199, 1616, $1590 \mathrm{~cm}^{-1}$; HRCIMS m/z calcd for $\mathrm{C}_{27} \mathrm{H}_{23} \mathrm{~N}_{3} \mathrm{O}+\mathrm{Na}^{+}(\mathrm{M}+$ $\mathrm{Na}^{+}$) 428.1739, found 428.1714 .

$N$-Methyl- $N$-phenyl-4-(2-methoxyphenyl)- $\beta$-carboline-3-carboxamide (10c). This compound, prepared from $7 \mathbf{c}$ (45 $\mathrm{mg}, 0.09 \mathrm{mmol})$ using the above general procedure, was obtained as an offwhite solid (33 mg, $0.08 \mathrm{mmol}, 92 \%$ ), $\mathrm{mp}>300^{\circ} \mathrm{C}:{ }^{1} \mathrm{H}$ NMR (DMSO-d 6 , $\left.300 \mathrm{MHz}\right) \delta 3.3$ (s, 3H), 3.5 (s, 3H), 6.67 (d, 1H, J = 8.2 Hz), 6.87 (d, 1H, J = $7.8 \mathrm{~Hz}), 7.0$ (m, 2H), 7.15 (m, 3H), 7.40 (m, 3H), 7.5-7.6 (m, 2H), 8.03 (d, 1H, J = $8.5 \mathrm{~Hz}), 9.2(\mathrm{~s}, 1 \mathrm{H}), 11.9(\mathrm{~s}, 1 \mathrm{H}) ;{ }^{13} \mathrm{C}$ NMR (DMSO-d $\left.\mathrm{d}_{6}, 75 \mathrm{MHz}\right) \delta 37.5,38.6,55.1,112.1,115.9,121.7,123.7,124.1,124.7,127.6$ (2C), 127.8, 127.9, 129.6 (2C), 129.9, 130.0, 131.1, 133.5, 133.8, 133.9, 135.6, 143.4, 158.7, 166.2, 170.2; IR (film) 3244, 1638, $1590 \mathrm{~cm}^{-1}$; HRCIMS $\mathrm{m} / z$ calcd for $\mathrm{C}_{26} \mathrm{H}_{21} \mathrm{~N}_{3} \mathrm{O}_{2}+\mathrm{Na}^{+}\left(\mathrm{M}+\mathrm{Na}^{+}\right)$ 430.1531, found 430.1503 .

$N$-Methyl- $N$-phenyl-4-(2-fluorophenyl)- $\beta$-carboline-3-carboxamide (10d). This compound, prepared from 7d (35 $\mathrm{mg}, 0.07 \mathrm{mmol}$ ) using the above general procedure, was obtained as an off-white solid (25 mg, $0.06 \mathrm{mmol}, 90 \%$ ), mp 127-129 ${ }^{\circ} \mathrm{C}$ : ${ }^{1} \mathrm{H}$ NMR (DMSO-d 6 , $300 \mathrm{MHz}$ ) $\delta$ 3.43 (s, 3H), 7.0-7.10 (m, 2H), 7.14 (m, 5H), 7.19 (d, 1H, J = 7.2 Hz), 7.26 (t, 2H, J = 8.4 Hz), 7.57 (d, 1H, J = $7.8 \mathrm{~Hz}$ ), 8.26 (d, 1H, J = $7.8 \mathrm{~Hz}$ ), 8.39 (m, 1H), 8.55 (s, $1 \mathrm{H}), 11.7$ (s, $1 \mathrm{H}) ;{ }^{13} \mathrm{C}$ NMR (DMSO-d 6 , 75 MHz) $\delta 38.9,116.3,116.5,117.2,117.8,123.5,123.7,124.5,124.9,126.1$, 127.3 (2C), 128.5, 128.8 (2C), 129.8, 129.1, 130.0, 130.6, 131.4, 131.5, 132.7, 136.0, 136.4, 
143.7, 148.2, 170.0, 168,2; IR (film): 3258, 1618, $1591 \mathrm{~cm}^{-1}$; HRCIMS $\mathrm{m} / \mathrm{z}$ calcd for $\mathrm{C}_{25} \mathrm{H}_{18} \mathrm{~N}_{3} \mathrm{OF}+\mathrm{Na}^{+}\left(\mathrm{M}+\mathrm{Na}^{+}\right)$418.1332, found 418.1339.

$N$-Methyl- $N$-phenyl-4-(3,4,5-trimethoxyphenyl)- $\beta$-carboline-3-carboxamide $\quad$ (10e). This compound, prepared from $7 \mathbf{e}(45 \mathrm{mg}, 0.08 \mathrm{mmol})$ using the above general procedure, was obtained as a white powder (31 mg, $0.07 \mathrm{mmol}, 84 \%$ ), mp $>300^{\circ} \mathrm{C}:{ }^{1} \mathrm{H}$ NMR (DMSO-d 6 , 400 MHz) $\delta 3.3$ (s, 3H), 3.8 (s, 6H), 3.9 (s, 3H), 6.42 (s, 2H), 6.75 (d, 1H, J = 7.58 Hz), 7.10 (t, 2H, J $=7.5 \mathrm{~Hz}$ ), $7.29(\mathrm{t}, 1 \mathrm{H}, \mathrm{J}=7.4 \mathrm{~Hz}), 7.56(\mathrm{t}, 1 \mathrm{H}, \mathrm{J}=7.4 \mathrm{~Hz}), 7.6(\mathrm{t}, 1 \mathrm{H}, \mathrm{J}=7.3 \mathrm{~Hz}), 7.7(\mathrm{~d}, 1 \mathrm{H}, \mathrm{J}=$ $8.4 \mathrm{~Hz}$ ), 8.18 (d, 1H, J = 8.0 Hz), $9.38(\mathrm{~s}, 1 \mathrm{H}), 11.2(\mathrm{~s}, 1 \mathrm{H}) ;{ }^{13} \mathrm{C}$ NMR (DMSO-d 6 , $\left.75 \mathrm{MHz}\right) \delta$ 35.5, 55.1, 57.3 (2C), 106.2 (2C), 114.4, 117.9, 120.8, 122.6, 124.2, 125.5, 126.4, 127.9 (2C), 128.2, 128.4, 128.9 (2C), 129.3, 131.4, 131.7, 134.3, 139.0, 153.0 (2C), 154.7, 165.5; IR (film): 3198, 1615, $1592 \mathrm{~cm}^{-1}$; HRCIMS $\mathrm{m} / \mathrm{z}$ calcd for $\mathrm{C}_{28} \mathrm{H}_{25} \mathrm{~N}_{3} \mathrm{O}_{4}+\mathrm{Na}^{+}\left(\mathrm{M}+\mathrm{Na}^{+}\right) 490.1743$, found 490.1754 .

\section{Acknowledgements}

The authors are grateful to the ICSN for a graduate fellowship (LXM). We thank Dr. Philippe Dauban for many helpful discussions and suggestions.

\section{References and Notes}

1. (a) Takayama, H.; Kurihara, M.; Kitajima, M.; Said, I. M.; Aimi, N. Tetrahedron 2000, 56, 3145. (b) Murakami, C.; Fukamiya, N.; Tamura, S.; Okano, M.; Bastow, K. F.; Lee, K. H.; Tokuda, H.; Mukainaka, T.; Nishino, H.; Lee, K.-H. Bioorg. Med. Chem. 2004, 12, 4963.

2. (a) Baruah, B.; Dasu, K.; Vaitilingam, B.; Mamnoor, P.; Venkata, P. P.; Rajagopol, S.; Yeleswarapu, K. R. Bioorg. Med. Chem. 2004, 12, 1991. (b) Rinner, U.; Hudlicky, T.; Gordon, H.; Pettit, G. R. Angew. Chem., Int. Ed. 2004, 43, 5342. (c) Boursereau, Y.; Coldham, I. Bioorg. Med. Chem. Lett. 2004, 14, 5841. (d) Cao, R.; Peng, W.; Chen, H.; Hou, X.; Guan, H.; Chen, Q.; Ma, Y.; Xu, A. Eur. J. Med. Chem. 2005, 40, 249. (e) Takasu, K.; Shimogama, T.; Saiin, C.; Kim, H.-S.; Wataya, Y.; Ihara, M. Bioorg. Med. Chem. Lett. 2004, 14, 1689.

3. For reviews, see: (a) Gardner, C. R.; Tully, W. R.; Hedgecock, C. J. Prog. Neurobiol. 1993,40, 1. (b) Dodd, R. H. Eur. Bull. Cogn. Psychol. 1992, 12, 484.

4. (a) Wang, Q.; Han, Y.; Xue, H. CNS Drug Rev. 1999, 5, 125. (b) Hollinshead, S. P.; Trudell, M. L.; Skolnick, P.; Cook, J. M. J. Med. Chem. 1990, 33, 1062. (c) Allen, M. S.; Tan, Y. C.; Trudell, M. L.; Narayanan, K.; Schindler, L. R.; Martin, L. R.; Schultz, C.; Hagen, T. J.; Koehler, K. F.; Codding, P. W.; Skolnick, P.; Cook, J. M. J. Med. Chem. 1990, 33, 2343.

5. Dorey, G.; Dubois, L.; Prado de Carvalho, L.; Potier, P.; Dodd, R. H. J. Med. Chem. 1995, 38, 189. 
6. Mehta, A.; Dodd R. H. J. Org. Chem. 1993, 58, 7587.

7. Batch, A.; Dodd, R. H. J. Org. Chem. 1998, 63, 872.

8. Batch, A.; Dodd, R. H. Heterocycles, 1999, 50, 875.

9. For reviews concerning $o$ - directed metalation of heterocyclic systems, see: (a) Quéguiner, G.; Marsais, F.; Snieckus, V.; Epsztajn, J. Adv. Heterocycl. Chem. 1991, 52, 187. (b) Snieckus, V. Chem. Rev. 1990, 90, 879.

10. Wagaw, S.; Buchwald, S. L. J. Org. Chem. 1996, 61, 7240.

11. For reviews, see: Miyaura, N.; Suzuki, A. Chem. Rev. 1995, 95, 2457. (b) Tietze, L. F.; Ila, H.; Bell, H. P. Chem. Rev. 2004, 104, 3453.

12. Broutin, P.-E.; Colobert, F. Eur. J. Org. Chem. 2005, 1113.

13. Largeron, M.; Farrel, B.; Rousseau, J.-F.; Fleury, M.-B.; Potier, P.; Dodd, R. H. Tetrahedron Lett. 2000, 41, 9403. 